\title{
Motivating and Enabling Organizational Memory with a Workgroup Wiki
}

\author{
School of Information, University of Michigan \\ 1075 Beal Ave \\ Ann Arbor, MI 48103 \\ samunson@umich.edu
}

Sean A. Munson

\author{
The Boeing Company \\ PO Box 3707 MC OF-LA \\ Seattle, WA 98124-2207 \\ sean.a.munson@boeing.com
}

\begin{abstract}
Workgroups can struggle with remembering past projects and sharing this information with other groups in the organization. In a case study of the deployment of MediaWiki as a publishing tool for building organizational memory, group members' motivation to document past projects increased. A browsable collection of past projects allowed for discovery of past work, building the reputation of individuals and the workgroup, and development of transactive memory within the workgroup. The "anyone can edit" feature, frequently touted as the main benefit of wikis, had both benefits and drawbacks in this implementation. Group members did not feel comfortable making substantial edits to others' content but did occasionally use the wiki to coauthor content and also categorize and link to others' content and fix typos, particularly when asked to help.
\end{abstract}

\section{Categories and Subject Descriptors}

K.4.3 [Computer and Society]: Organizational Impacts - computer supported cooperative work.

\section{General Terms}

Management, Documentation, Design

\section{Keywords}

Organizations, knowledge transfer, organizational memory, wikis, repositories, knowledge exchange

\section{INTRODUCTION}

Many firms are turning to electronic knowledge repositories and other tools for improving information reuse and identifying experts within the organization. This work presents findings from a study of the deployment of a wiki as workgroup knowledge repository, with the specific goal of improving organizational memory within the workgroup and making the group's previous work more visible to other groups in the company. These findings are:

- Providing employees with a space to publish their past work in a way that they believe will lead to reuse motivates employees to document their work.

- A collection of linked project, process, and user pages enables improved search and browsing, increases potential for group

Permission to make digital or hard copies of all or part of this work for personal or classroom use is granted without fee provided that copies are not made or distributed for profit or commercial advantage and that copies bear this notice and the full citation on the first page. To copy otherwise, or republish, to post on servers or to redistribute to lists, requires prior specific permission and/or a fee.

WikiSym '08, September 8-10, Porto, Portugal.

Copvright 2008 ACM 978-1-60558-128-3/08/09...\$5.00. and individual reputation management, and raises awareness of group members' expertise over individual archives or unlinked files. Scoping this collection at the workgroup level was effective for the field site.

- The "anyone can edit" feature of wikis, popularly described as their primary benefit [e.g. 13], has both costs and benefits in this setting. Group members do not want to change content they perceive as belonging to others, and do not want others to change content they feel belongs to them. Editing is more beneficial for occasionally co-authoring content, categorizing and linking others' content, and correcting typos.

Before discussing these findings, I begin this paper with a review of organizational memory, the use of wikis as repositories, and user motivations for contribution. Next, I describe the field study site and methods and finally consider the results of the study.

\section{ORGANIZATIONAL MEMORY AND KNOWLEDGE REPOSITORIES}

Organizations are more capable of solving current problems if they can quickly retrieve past solutions and locate experts among their ranks $[1,14]$. In organizations, some past solutions and justifications may already be documented, while some may remain tacit. Gorman notes that making tacit knowledge explicit can facilitate technology transfer, though this is rarely sufficient on its own [3]. Transactive memory, the combination of a group's knowledge and awareness of individuals' knowledge and skills, helps teams to coordinate and apply individual expertise to problems [16].

Electronic knowledge repositories are one tool for developing organizational memory $[4,7,8]$. Previous work describes wikis as a "midweight" tool for collaborative memory [17] that offers easy access and requires low effort to contribute content while not requiring users to conform to a rigid, formal structure. Maxwell evaluates wikis as a publishing platform and notes that wikis may be an appropriate alternative to more rigid content management systems, especially because they allow contributors and editors to define the structure rather than having a structure defined by the tool or a technical team [9]. Others note the value of wikis as a collaborative tool for consensus building, knowledge co-creation, and sharing of different viewpoints $[6,8]$.

Much of the literature on knowledge repositories has been concerned with the challenge of motivating contributions $[5,6,7,15]$. Previously identified motivators to contribute include economic rewards $[5,7]$, increased access to information / reciprocity $[5,7]$, career advancement or security $[5,7]$, enhanced reputation $[5,8]$, personal satisfaction [13], enjoyment of helping others [7], knowledge self-efficacy [7], process improvement [8], and making work easier [8]. 




Figure 1. Wiki growth, by characters and pages. Introduced to group at $\mathrm{A}$; wiki party at $\mathrm{B}$; past patents begin to be added at C; WYSIWYG editor added at $D$.

Orlikowski found that employees could be reluctant to contribute to knowledge repositories if they fear a loss of knowledge power [12], though Kankanhalli et al did not find this in their study. Finally, trust also influences contributions to electronic knowledge repositories. In organizations with more trust between employees, workers were less likely to perceive the effort required to add the information to the repository as a barrier to contribution [7].

\section{PAYLOADS STUDIO FIELD STUDY}

Within Boeing, the Payloads Studio explores advanced concepts for the future of commercial aircraft interiors. The 27 members of the group work in an offsite location in an open-plan office. Despite successes, members of the group report difficulty in communicating and transitioning their work to production groups. Group members also sometimes felt that project documentation was unlikely to be reused in the future, and so were not motivated to provide it.

By summer 2007, members of the Payloads Studio wanted a project repository that employees in other groups could browse. This repository would also serve as a record of past projects for future use by the group. The group members felt that a wiki would meet these needs. Within the workgroup, other software tools are used to support project management (a group file server and Microsoft SharePoint sites for each project) and sharing of group information such as vacation schedules, conference reports, work processes, how-tos, and information on recording projects in the time keeping system (the group file server).

This study discusses the adoption and first five months of wiki use by the workgroup. Perceived and anticipated benefits were the primary consideration for group members' decisions about how to use the wiki during this time, and future use of the wiki will likely be affected by the extent to which these expectations are realized.

\section{METHODS}

To study wiki deployment at the Payloads Studio, three types of data were collected: analysis of edit logs, interviews, and content analysis of wiki pages. Edit logs were captured and analyzed from November 2007 - April 2008. Wiki pages were coded according to their subject, and summary statistics about page length and edit count were prepared.

In addition to monitoring wiki content, a purposive sample of seven members of the group participated in semi-structured interviews about their expectations and use (or non-use) of the wiki.

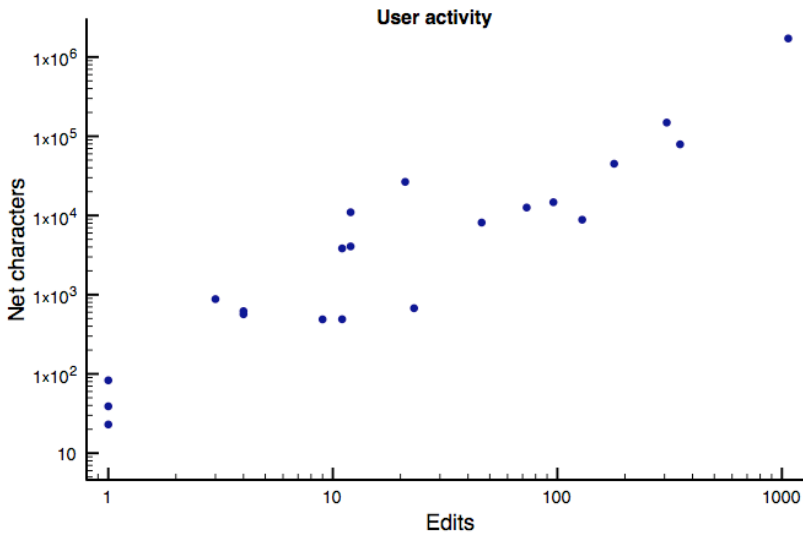

Figure 2. Wiki editors by activity (edits and net characters)

These users included a range of group members from someone who had not contributed through heavy contributors. The interview data were coded both deductively - corresponding to constructs identified through review of previous work - and inductively to identify emergent themes. During the interviews, I was able to use the edit $\log$ s to ask about specific contributions the interviewee had made. The goal was to triangulate data from the three sources to view the themes from different perspectives.

The author was employed by Boeing during this study. Another group member was the wiki's champion and primary advocate, but the author was responsible for the installing the wiki and answering users' technical questions about tool. This may have affected what workgroup members chose to disclose during interviews.

\section{FINDINGS AND DISCUSSION}

The wiki team selected and deployed MediaWiki [10] as the group's repository. In informal conversations with group members, they indicated they were familiar with Wikipedia's browsing interface and tone, which led to the selection of MediaWiki software. The wiki was initially introduced to three members of the workgroup who seeded content about three past projects. On 15 November, the wiki's champion unveiled the system to the rest of the Payloads Studio; at the conclusion of the meeting each team member received an email with instructions for signing up and creating an account

One month later, the wiki champion organized a "wiki party," a work session with snacks, during which group members were encouraged to add information about past projects to the wiki. Team members familiar with MediaWiki were on-hand to help others enter content. The work session was also an opportunity for the group members to develop norms and policies regarding use of the wiki. Questions such as "when should we add content to the wiki?," "what should I add to the wiki?" and "when do we use the wiki instead of SharePoint?" were asked throughout the session. None of these questions was definitively answered, but the asking and subsequent discussion made group members more aware of each others' opinions and expectations. During the work session and subsequent conversations, it also became apparent that learning wiki syntax was a substantial barrier to entering more complex content into the system, and users requested a WYSIWYG editor [11], which was added on 23 January.

As of 3 April 2008, the wiki had 762 pages (excluding help pages), edited by a mean of 1.17 users per page. 21 of the group's 
27 members had made wiki edits; 12 contributed 3,000 characters or more; the next highest contributor added 882 characters. The wiki's growth is shown in figure 1, with user contributions in figure 2 .

\subsection{Content}

Table 1 presents a summary of wiki content. Aside from category pages (which support navigation) and uploaded files (that are used to illustrate and provide further depth for pages in the other categories), the largest content category is an archive of past patent filings (139 pages), created almost entirely by one group member. Project information, the wiki's primary initial purpose, accounts for 106 pages. Group members expanded their wiki use to include information related to work practice ( 8 pages), such as codes to annotate time sheets and the group's process for intellectual property disclosures and securing proprietary information agreements. Prior to the wiki, this information was either located in Microsoft Word, Excel, or PowerPoint documents on a file server or group members' hard drives or existed only with its creator.

16 of the 27 group members created user pages for their wiki account. Most of these profiles contain contact information, a professional headshot of the group member, and links to their past projects. The first group member to create a user profile describes her reasons: "I think sometimes that it really helps to put a name and face with the work, and to let people see who we are as a group. We don't really have an org chart (in our group), but this way people can see what we each do." A smaller number of group members $(n=3)$ have built more elaborate profiles, including information such as a list of their patent applications, educational and employment history, hobbies, and work interests, and a listing of what they consider their areas of expertise. Another group member said he is considering using his wiki user profile as a personal portfolio manager. He believes this content can build his reputation within the company, supporting his application for a technical fellow role within the company and helping others find him to ask questions about topics on which he is an expert.

\subsection{Motivation to contribute}

For contributing past project information, group members interviewed were primarily motivated by wanting others to be able to reuse their work in the future $(n=6)$. Group members felt frustrated that their and others' past work would just "fade away" and that they did not want people to "redo everything [they] did two years from now." One user described her motivations: "I wanted it to fill the giant hole we've been missing with all our projects, learnings, and money spent that currently either leaves with people or disperses into the air."

Most group members interviewed $(n=6)$ felt that sharing project information on the wiki increased their influence and power in the organization, both across time and across organizational boundaries, and said that the wiki made them more motivated to document projects than in the past. One group member indicated that she was concerned about adding contact information for collaborators outside of the company - not out of fear of losing her influence or being left out of an important conversation, but out of concern for the contacts being over utilized. After some deliberation, she decided to include both the contacts and a note asking readers to email her before contacting them.

Three interviewees had shared project reports outside of the group using the wiki. They commented that sharing a link over email is less imposing than emailing someone a large file and that recipi-

\begin{tabular}{|c|c|c|c|c|}
\hline \multirow{2}{*}{ Page type } & \multirow{2}{*}{ Pages } & \multicolumn{3}{|c|}{ Mean per page } \\
& & Edits & Editors & Chars \\
\hline Project pages & 106 & 7.2 & 1.3 & 4219 \\
\hline Processes & 8 & 6.3 & 1.4 & 1828 \\
\hline Patent pages & 139 & 3.0 & 1.0 & 11276 \\
\hline Industry data & 4 & 3.0 & 1.3 & 2661 \\
\hline User pages & 24 & 13.4 & 1.5 & 727 \\
\hline Categories & 154 & 1.2 & 1.0 & 49 \\
\hline Indices \& templates & 17 & 12.2 & 3.1 & 1280 \\
\hline Images and files & 310 & 1.3 & 1.1 & 17 \\
\hline
\end{tabular}

Table 1. Wiki content as of 3 April 2008. User pages include one redirect and eight profiles that one user created to provide information about past members of the group.

ents seemed very receptive to receiving a link rather than a PDF or Microsoft Word report. For the same reasons, they said that sending links seemed to increase the chances that others would forward their reports.

The interviewed group members feel that all of the past projects should eventually be on the wiki, though only about $30 \%$ of past projects currently have wiki pages. One of the challenges has been that very few of the projects had documentation that could simply be copied over to the wiki - indeed, every project added to the wiki so far has required writing at least some new content. The easiest projects to add tend to be recently completed projects. For these, the project team members are still in the group, the project information is still fresh in their minds, and they are still able to quickly access any of the documents produced while working on the project. Documentation on the wiki has also become a formal requirement for closing out projects.

Older projects are more difficult to add, and while there is management support for this activity, it is not a requirement. Group members gave different examples of how they chose which older projects to add, including selecting projects of which they are particularly proud, projects they often get questions about, or projects that they run across when cleaning up the file server, SharePoint, or their computers.

Users felt challenged by uncertainty about which content, and how much content, they should add. Some preferred an archiveeverything approach; others tried to only add content that they felt was likely to be reused. There was some tension between group members with different documentation styles. Those who prefer to document everything expressed concern that others' entries were incomplete, and worried that potentially information was being lost. In contrast, users who tried to document only information they felt would be reused were concerned that some others' pages might be "much too detailed for someone who just finds it from a search, maybe even intimidating." Most interviewees who voiced this concern felt that the different documentation styles will converge as the group gains experience with the wiki.

Interviewees who added group process information $(n=3)$ were also motivated by wanting to record information that might otherwise be lost or difficult to find. They say that they have to answer fewer questions about where to find information they have 
placed on the wiki than about information they store on the file server. Keeping frequently accessed group information (forms, job numbers, etc.) alongside the archived projects also helps bring team members coming back to the wiki during their day-to-day activities. This avoids the common groupware pitfall where new tools may fall outside of, or require changes to, normal group processes and are subsequently neglected by potential users [10].

Other than the challenge of finding time to contribute and the difficulty of locating information about some past projects, technical and interface difficulties were the most frequently mentioned barriers to contribution. These included slow server response time $(n=6)$, difficulty learning wiki syntax $(n=3)$, and a file upload interface that is focused on images $(n=2)$. Additional technical issues observed but not mentioned included at least 11 instances of duplicate pages or file uploads, resulting from confusion with MediaWiki's case sensitivity for page names.

\subsection{The value of a collection}

Most interviewed group members favored a combination of the wiki and file server $(n=2)$ or strongly preferred the wiki to the file server and SharePoint sites $(n=4)$. "The wiki is head and shoulders above all other storing and sharing tools we are currently using." Many reasons for this preference related to the wiki being a collection of pages about several projects, processes, and group members: a collection enables one-stop searching and browsing across all of its content, content creators can use other pages in the collection as examples they can imitate, and collection offers a better view of a project or team member in the context of the group's full body of work.

Group members said they found the wiki particularly useful when looking for information on past projects to apply to their current work. Links that spanned different types of content helped them identify related projects they would not have known to include in their search, and through these links some discovered projects completed before they joined the group or that they had forgotten. Interviewees reported that including findings from past projects in current work helped them to find new and better solutions and gave the current work greater legitimacy when presented to the others. Some interviewees - particularly the newer members of the group - said that they would enjoy browsing past projects, but felt constrained by time and mostly used the wiki when there was a specific need for a current project.

All interviewees also said it was easier to search for information on the wiki than on the file server or SharePoint. Users feel constrained by the file organization scheme on these tools, and this hierarchy, they say, leaves information "buried." In contrast, the wiki allows users to define their own links between the wiki content. Archiving this information on a single wiki gives users a "one stop shop" in contrast to "having to hunt around" different folders or projects for data. This type of central collection, with multiple link paths to content and improved full-text search can help avoid information retrieval problems that arise when users categorize content differently [2].

The combination of improved search and browsability of the wiki collection leads group members to anticipate the content they add to the wiki being discovered by others in the company. In the words of one group member, "I am looking forward to the day when I get a phone call from a random person saying they found something interesting on the wiki and want to know more about it.”
A collection also facilitates comparison between different project reports; three of the interviewees described browsing between their write-ups and others' work for ideas about how to improve their documentation. Early in the wiki's deployment, there had been much discussion about whether or not some group members should be assigned to create a template for project documentation. Two users drafted pages with sample headings that could be copied into new project pages, but contributors instead prefer to use others' project documentation as inspiration for what they choose to include in the projects they document and how they choose to organize them.

Finally, the ability to navigate between user profiles, projects, processes, and group information, all with clear authorship, supports the development of transactive memory within the group. Two of the interviewees - one newer and one more experienced member of the group - mentioned learning more about other group members' involvement with past projects as a specific a motivator for browsing the wiki, and two more mentioned an increased awareness of what others were working on as a result of visiting the wiki. The group members are aware of this benefit; helping others' in the company find their work and recognize their expertise and experience has been the primary motivator for writing user profiles. "If someone reads about [a project] and notices that I worked on it, that's good, but if they click on my name, or on another link, and see that I worked on [several projects on a similar topic], that's better. I think they'll see this is something I'm interested in and good at." If use of the group wiki had involved more knowledge synthesis, the resulting suppression of individual authorship may have stifled the development of transactive memory.

While no new members entered the workgroup during the study period, I speculate that the collection may be of particular value in orienting newcomers to the group, both by helping them discover and learn about past projects and as well as their coworkers' expertise and experience.

\subsection{Anyone can edit}

Of the 24 user pages, 12 were edited by two users, all of which were typo corrections or the addition of the page to a category. Other pages with multiple editors ( $\mathrm{n}=45$; excluding uploaded images, categories, and user pages) tended to be index pages $(n=9)$, pages on which additional editors only fixed typos or categorized the page $(n=20)$ or pages where the primary author asked a user more experienced with MediaWiki syntax for assistance with formatting content $(n=12)$. Three of the remaining four pages were from one project with two of the project's team members coauthoring pages. Other than index or category pages, five users contributed to or edited pages for which they were not the primary author (i.e., pages to which they did not contribute $>80 \%$ of the character count). Each of these five users is also a content contributor; edits to pages they started outnumber edits to pages others have started.

In contrast with previous research on corporate wikis [8], the very limited synthesis and editing of others' content on this wiki leaves little differentiation in user roles. Three users were more active editors of the index pages (e.g. listings of past projects), adding links to pages that they hoped others would write. Beyond the differences in quantity of contributions, this seeding of the wiki with references to future pages was the only behavior that clearly distinguished types of contributions. 
Three interviewees indicated that they have had ideas about improving pages that others have authored, but saw those pages as "their content" and "did not want to mess with it." Instead, they make these suggestions verbally or by email rather than making changes themselves. "I don't believe people should edit other peoples' work. If you have a comment, write a comment. Don't edit someone else's work." While users are aware of MediaWiki's discussion pages, they have not used them. Interviewees instead preferred to directly email or talk with the page's author.

Users were similarly uncomfortable having others edit their content; one user described being "taken aback" by another user's reorganization of some content she had contributed. Another user wanted ways to signal that she considered a page final. "You wouldn't want to lock people out [from editing], just a way to tell people" that the document has been finalized. This user sees her contributions to the wiki - which have been shared with others in the company - as shaping her reputation within the company, and says that she wants them to reflect "my conclusions." The resistance to having others edit the content affects both content and presentation. "If you've gone to the trouble of wordsmithing your content to say just what you mean just how you want, someone coming in and changing it wouldn't be so good" says another group member. Even when team members coauthor a page, they talk about "dividing up" and "owning" different sections.

In this implementation, the group uses the wiki more as a publishing-oriented content management system rather than as a space for knowledge co-creation or synthesis. Group members add content near or after the conclusion of a project, and see this information as archival. The collaborative editing features do still offer benefits for users: they can get help from other users, they can fix typos they see, and they can make links between different pieces of content.

\section{CONCLUSIONS}

The group's experience with the wiki suggests that a wiki can meet a workgroup's needs for a publishing system to archive and share past project information and group resources. Tactics such as face-to-face group work sessions with experienced team members on hand to help overcome technical issues can ease adoption issues and give group members a chance to begin developing norms for how the tool will be used.

Providing employees with a browsable space where they can share past work with others in the company increased group members' motivations to document this work. The additional costs of documenting projects on the wiki were outweighed by anticipated benefits. These perceived benefits include managing one's reputation within the company, increasing the influence of one's work, and avoiding future duplication of efforts. Similarly, for the interviewees, the cost of adding and formatting group information for the wiki is outweighed by the perceived benefits of helping others in the group quickly locate and access this information. It is not clear whether these perceived benefits will be realized in practice, but the ease of public access made such benefits plausible and motivating to the workgroup members.

Group members were uncomfortable with editing content they perceived as belonging to others. At the same time, the anyonecan-edit feature has allowed group members to help categorize others' content, fix typos, and respond to requests for help with wiki syntax and formatting. Two group members have used the wiki to collaboratively author a report, and another of the interviewed group members liked having that potential. Despite not using the wiki for informal synthesis, these other examples of using the software as a collaborative editing tool suggest that the choice of a wiki over other content management systems is still beneficial.

\section{ACKNOWLEDGEMENTS}

Thanks to Beth Yakel, Mark Ackerman, Paul Resnick, Xiaomu Zhou, and anonymous reviewers who provided comments on earlier versions of this paper. I also thank the members of the Payloads Studio who participated in the interviews.

\section{REFERENCES}

[1] Ackerman, M. 1998. "Augmenting Organizational Memory: A Field Study of Answer Garden," ACM Transactions on Information Systems 16(3): 203-224.

[2] Berlin, LM; Jeffries, R; O’Day, VL, Paepcke, A \& Wharton, C. 1993. "Where Did You Put It? Issues in the Design and Use of a Group Memory," Proceedings of ACM INTERCHI '93: 23-30.

[3] Gorman, ME. 2002. "Types of Knowledge and Their Roles in Technology Transfer," Journal of Technology Transfer 27: 219-231.

[4] Grudin, J. 1994. "Groupware and Social Dynamics: Eight Challenges for Developers," Communications of the ACM 37(1): 92-105.

[5] Hall, H. 2001. "Social Exchange for knowledge exchange," Managing Knowledge: conversations and critiques, 10-11 April 2001.

[6] Hasan, H; Meloche, JA; Pfaff, CC; Willis D. 2007. "Beyond Ubiquity: Co-Creating Knowledge with a Wiki," UBICOMM07.

[7] Kankanhalli, A; Tan, BCY; Wei, K. 2005. "Contribution Knowledge to Electronic Knowledge Repositories: An Empirical Investigation,” MIS Quarterly 29(1): 113-143.

[8] Majchrzak, A; Wagner, C; and Yates, D. 2006. "Corporate Wiki Users: Results of a Survey," WikiSym '06.

[9] Maxwell, JM. 2007. "Using Wiki as a Multi-Mode Publishing Platform,” SIGDOC '07.

[10] MediaWiki. http://www.mediawiki.org

[11] MediaWiki+FCKeditor. http://mediawiki.fckeditor.net.

[12] Orlikowski, WJ. 1992. "Learning from Notes: Organizational Issues in Groupware Implementation," CSCW 1992.

[13] Taylor, C. 2005. "It's a Wiki, Wiki World," Time.

[14] Walsh, JP and Ungson, GR. 2008. "Organizational Memory," The Academy of Management Review 16(1): 57-91.

[15] Watson, S and Hewett, K. 2006. "A Multi-theoretical model of Knowledge Transfer in Organizations: Determinants of Knowledge Contribution and Knowledge Reuse," Journal of Management Studies 43:2.

[16] Wegner, DM. 1986. "Transactive memory: A contemporary analysis of the group mind." In MB \& GGR (Eds.), Theories of Group Behavior (pp. 185-205). New York: SpringerVerlag.

[17] White, KF and Lutters, WG. 2007. "Midweight Collaborative Remembering: Wikis in the Workplace," CHIMIT ' 07. 